\title{
Some Results on Riemannean Multiple Barycenters
}

\author{
Mostafa Zahri*, Naji Khallaf \\ Department of Mathematics, College of Science, Taibah University, Madinah, Saudi Arabia \\ Email: zahri@gmx.net, drnaji123@yahoo.com
}

Received 11 March 2014; revised 11 April 2014; accepted 18 April 2014

Copyright (C) 2014 by authors and Scientific Research Publishing Inc.

This work is licensed under the Creative Commons Attribution International License (CC BY). http://creativecommons.org/licenses/by/4.0/

cC) (i) Open Access

\begin{abstract}
A characteristic of a special case of Riemannean barycenters on the unit circle is presented. The non-uniqueness of such barycenters leads to an interesting study of the so-called multiple barycenters. In this work, we deal with a smooth one-dimensional manifold $S^{1}$ only. Some theoretical and computational analysis is listed.
\end{abstract}

\section{Keywords}

Smooth Manifold, Geodesic, Barycenter of Masses, Metric Space, Multiple Barycenters

\section{Introduction}

The Riemanean geometry is the study of curved surfaces. One might have a cylinder, or a sphere. For instance, one can use a cardboard paper towel roll to study a cylinder and a globe to study a sphere. In such spaces, the shortest curve between any pair of mass points on such curved surface is called a minimal geodesic or geodesic. One can find a geodesic between two mass points by stretching a rubber band between them. The first thing that will be noticed is that sometimes there is more than one minimal geodesic between two mass points. For instance, there are many minimal geodesics between the north and south poles of a sphere. This leads to derive more generalized formulas, which are true in the Euclidean space. Therefore, one of our main concern in this work is to motivate researcher for finding exact formulas for computing Barycenters on non-Euclidean spaces, namely on manifolds. In [1], the authors suggest an estimation of the so-called Riemannean Barycenters. For a use in the same context, we refer for instance to [2]. Where, in [3] Riemannian means were presented as solution of variational problems. We think that for special case, for instance smooth manifolds, we could derive similar formulas to the Euclidean case, define and study interesting properties of Riemannean Barycenters.

In this work, we begin by setting out some basic theory of the geodesic of the unit circle as one dimensional

\footnotetext{
${ }^{*}$ Corresponding author.
} 
manifold, we define the multiple Barycenters and compute it for some special cases. For more details, we refer to [4]-[6].

Let $I$ be an interval of real numbers. Then, a curve $\gamma$ is a continuous mapping given as $\gamma: I \rightarrow S^{1}$, where $S^{1}$ is a smooth Riemannean manifold. The curve $\gamma$ is said to be simple if it is injective. If $I$ is a closed bounded interval $[a, b]$, we also allow the possibility $\gamma(a)=\gamma(b)$ (this convention makes it possible to talk about closed simple curve). If $\gamma(x)=\gamma(y)$ for some $x \neq y$ (other than the extremities of $I$ ), then $\gamma(x)$ is called a double (or multiple) point of the curve. A curve $\gamma$ is said to be closed or a loop if $I=[a, b]$ and if $\gamma(a)=\gamma(b)$. A closed curve is thus a continuous mapping of the circle. Let $x, y$ are two points of $S^{1}$ subset of $R^{n}$. If $\gamma$ is a curve such that $\gamma(a)=x, \gamma(b)=y$ and $\gamma(t) \in S^{1} \quad \forall t \in[a, b]$, we said that $\gamma$ is a connection between $x$ and $y$. We define $C_{(x, y)}$ the set of all connections between $x$ and $y$ on $S^{1}$. Let $x, y$ be two mass points of $S^{1}$. The geodesic is the minimum distance between $x$ and $y$ on $S^{1}$ (or the shortest path), denoted by $g(x, y)$, which is given by $g(x, y):=\inf \left\{L_{\gamma} \mid \gamma \in C_{(x, y)}\right\}=L_{\gamma^{*}}$. We say that $X$ is a geodesic space if for any pair $x, y \in X$ there is a minimal geodesic from $x$ to $y$ and this is given as a minimal of $L_{\gamma}=\int_{x}^{y}|\mathrm{~d} \gamma(t)|$, where $\gamma(t)$ is a curve joining $x$ and $y$. Let two points $x, y \in S^{1} \subset R^{n}$. The geodesic (shortest path) between the two points $x$ and $y$ is given by $g(x, y)=\cos ^{-1}(\langle x, y\rangle)$, where $\langle x, y\rangle$, is the scalar product on $R^{n}$. The shortest geodesic (which is a metric on $S^{1}: t \rightarrow \gamma(t)$ that joins two non antipodal points. The geodesic distance between the two points is precisely equal to $\alpha$. A midpoint $m$ of two masses on $S^{1}$ is defined $g(x, m)=g(y, m)=\frac{1}{2} g(x, y)$, where $g$ is a geodesic on $S^{1}$. For more details, we refer to [7]-[9].

Example 1. Consider the following examples on the unit circle:

1) Each point on $S^{1}$ is midpoint of itself $g(x, m)=g(m, x)=\frac{1}{2} g(x, x)=0$.

2) The midpoint of $x=\mathrm{e}^{\mathrm{i} \frac{\pi}{2}}$ and $y=\mathrm{e}^{\mathrm{i} \pi}$ is the unique point $m=\mathrm{e}^{\mathrm{i} \frac{3 \pi}{4}}$, therefore

$$
\frac{\pi}{4}=g(x, m)=g(m, y)=\frac{1}{2} g(x, y)=\frac{1}{2} \frac{\pi}{2} .
$$

3) Observe that even if $\frac{\pi}{4}=g(x, m)=g(m, y) \neq \frac{1}{2} g(x, y)$, for $m=\mathrm{e}^{\mathrm{i} \frac{7 \pi}{4}}$. The fact that $g(x, y)=2 \pi-\frac{\pi}{2}$ is not a geodesic between $x$ and $y$.

4) The midpoint on $S^{1}$ is not unique: Consider $x=\mathrm{e}^{\mathrm{i} \frac{\pi}{2}}$ (north pole) and $x=\mathrm{e}^{\mathrm{i} \frac{3 \pi}{2}}$ (south pole),

$$
\pi=g(x, m)=g(m, y)=\frac{1}{2} g(x, y) \text { for } m=\mathrm{e}^{\mathrm{i} \pi} \text { and } x=\mathrm{e}^{\mathrm{i} 2 \pi} .
$$

\section{Barycenters on $S^{1}$}

The arithmetic mean of a finite set of mass points $x_{1}, \cdots, x_{n}$ the Euclidean space $R^{d}$. is the unique point $\mathrm{x}$ that minimizes the sum of the squared distances to the given mass point, that is $E(x)=\sum_{i=1}^{n} d^{2}\left(x, x_{i}\right)$, where $d(\cdot, \cdot)$ is an Euclidean distance in $R^{d}$. The point realizing the minimum of the function $E(x)$ is called also Barycenter of the set of mass points $\left(x_{i}\right)_{1 \leq i \leq n}$. In this section we would like to extend this definition to a smooth Riemannean manifold $S^{1}$, and for more information, we refer to [1] [3].

Definition 1. The Barycenter of the set of mass points $x_{1}, \cdots, x_{n}$ subset $S^{1}$ with weighs $w_{1}, \cdots, w_{n} \geq 0$ (respectively) is the mass point minimizing the following functional (Energy function):

$$
E(x)=\sum_{i=1}^{n} w_{i} g^{2}\left(x, x_{i}\right)=\int_{S^{1}} g^{2}(x, y) \mu(\mathrm{d} y)
$$

where $g(\cdot, \cdot)$ is the geodesic in $S^{1}$ and $\mu$ is the distribution in the continuous case. 
Lemma 1. Let $S^{1}$ and $x_{1}, x_{2} \in S^{1}$ are two mass points with weights $w_{1}$ and $w_{2}$, such that $g\left(x_{1}, x_{2}\right)=L_{\gamma}$ with $\gamma:[0,1] \rightarrow S^{1}, \gamma(0)=x_{1}$ and $\gamma(0)=x_{2}$. If $x_{c}$ is the geodesic Barycenter of $x_{1}$ and $x_{2}$ in $S^{1}$, then there exists $t_{c} \in[0,1]$ such that $\gamma\left(t_{c}\right)=x_{c}$.

Proof. We prove that the geodesic Barycenter of two mass points is a mass point contained on the shortest path. We have

$$
\min _{x \in S^{1}}\left(w_{1} g^{2}\left(x_{1}, x\right)+w_{2} g^{2}\left(x_{2}, x\right)\right)=w_{1} g^{2}\left(x_{1}, x_{c}\right)+w_{2} g^{2}\left(x_{2}, x_{c}\right) .
$$

Suppose that $x_{c}$ is not element of $\gamma([0,1])$. There exists $t_{*} \in[0,1]$ such that

$$
\min _{t \in[0,1]} g\left(x_{c}, \gamma(t)\right)=\min _{t \in[0,1]} g\left(x_{c}, \gamma(t)\right)=g\left(x_{c}, \gamma\left(t_{*}\right)\right) .
$$

it follows

$$
g\left(x_{1}, \gamma\left(t_{*}\right)\right)+g\left(x_{2}, \gamma\left(t_{*}\right)\right) \leq g\left(x_{1}, x_{c}\right)+g\left(x_{2}, x_{c}\right),
$$

( $x_{c}$ is not element of $\gamma([0,1])$ ). It implies

$$
w_{1} g\left(x_{1}, \gamma\left(t_{*}\right)\right)+w_{2} g\left(x_{2}, \gamma\left(t_{*}\right)\right) \leq w_{1} g\left(x_{1}, x_{c}\right)+w_{2} g\left(x_{2}, x_{c}\right) .
$$

In fact, the point $x_{c}$ satisfies the minimal and the following equality $\gamma\left(t_{*}\right)=\gamma\left(t_{c}\right)=x_{c}$.

Lemma 2. Let two mass points $x_{1}, x_{2} \in S^{1}$ with weights $w_{1}$ and $w_{2}$ respectively. If for all $x, y \in \operatorname{conv}\left(x_{1}, x_{2}\right) ; g(x, y)$ are unique, then the geodesic Barycenter of $x_{1}, x_{2}$ is unique.

Proof. Suppose that the Barycenter is not unique, and denote by $x_{c}$ and $y_{c}$ solving the energy functional. From Lemma 1, we know that the geodesic Barycenter is contained on the shortest path between $x_{1}$ and $x_{2}$, it means

$$
g\left(x_{1}, x_{2}\right)=g\left(x_{1}, x_{c}\right)+g\left(x_{2}, x_{c}\right)=g\left(x_{1}, y_{c}\right)+g\left(x_{2}, y_{c}\right) .
$$

$x_{c} \neq y_{c}$ there exists two geodesics connecting $x_{1}$ to $x_{2}$, this contradicts the uniqueness of the geodesic.

Definition 2. Let an oriented unit circle with origin $x_{0}=(1,0) \in S^{1}$, and a subset of mass points points $\left(x_{i}\right)_{1 \leq i \leq n}$ subset on $S^{1}$. We denote by $w_{0}$ the number of all geodesics, their corresponding curves contain the origin $w_{0}=\#\left\{L_{\gamma_{i, j}}<\pi \mid i, j \in I, \exists t_{0}: \mathrm{e}^{i 0}=\gamma_{i, j}\left(t_{0}\right) \in \gamma_{i, j}(] 0,1[)\right\}$, where $I=1, \cdots, n$.

\section{Geodesic Multiple Barycenters on $S^{1}$}

The existence of Barycenters on $S^{1}$ interpreted as one dimensional Riemannean space does assure it uniqueness. In this section, we give a general condition of the existence of a geodesic multiple Barycenter on $S^{1}$.

Definition 3. (Geodesic Multiple Barycenter). We said that a set of mass points have a multiple Barycenter if the solution of the energy function is not unique.

Example 2.

1) The Barycenter of the north and south poles is not unique i.e. 0 and $\mathrm{e}^{\mathrm{i} \pi}$.

2) We examine a simple example on the unit circle. Compute the Barycenter of four mass points

$$
\begin{gathered}
X=\left\{0, \mathrm{e}^{\mathrm{i} \pi / 2}, \mathrm{e}^{\mathrm{i} \pi}, \mathrm{e}^{\mathrm{i} 3 \pi / 2}\right\} \\
\min _{x \in S^{1}} F(x):=\min _{x \in S^{1}}\left(g^{2}(x, 0)+g^{2}\left(x, \mathrm{e}^{\mathrm{i} \pi / 2}\right)+g^{2}\left(x, \mathrm{e}^{\mathrm{i} \pi}\right)+g^{2}\left(x, \mathrm{e}^{\mathrm{i} 3 \pi / 2}\right)\right) . \\
F(x):=g^{2}(x, 0)+g^{2}\left(x, \mathrm{e}^{\mathrm{i} \pi / 2}\right)+g^{2}\left(x, \mathrm{e}^{\mathrm{i} \pi}\right)+g^{2}\left(x, \mathrm{e}^{\mathrm{i} 3 \pi / 2}\right)
\end{gathered}
$$

Define $f(\arg (x)):=\arg (x)^{2}+(\arg (x)-\pi / 2)^{2}+(\arg (x)-\pi)^{2}+(\arg (x)-3 \pi / 2)^{2}$.

It implies

$$
f(z):=z^{2}+(z-\pi / 2)^{2}+(z-\pi)^{2}+(z-3 \pi / 2)^{2} .
$$


Differentiations with respect to $z$

$$
\frac{\partial f}{\partial z}(z):=2 z+2(z-\pi / 2)+2(z-\pi)+2(z-3 \pi / 2)=8 z-2 \pi
$$

1) $x \in S^{1}$ with $0 \leq \arg (x) \leq \pi / 2$ :

From $\frac{\partial f}{\partial z}(z)=0$ it follows $x=\mathrm{e}^{\mathrm{i} \pi / 4}$ and $F(x)=2\left((\pi / 4)^{2}+(3 \pi / 4)^{2}\right)$.

2) $x \in S^{1}$ with $\pi / 2 \leq \arg (x) \leq \pi$ :

From $\frac{\partial f}{\partial z}(z)=0$ it follows $x=\mathrm{e}^{\mathrm{i} 3 \pi / 4}$ and $F(x)=2\left((\pi / 4)^{2}+(3 \pi / 4)^{2}\right)$.

3) $x \in S^{1}$ with $\pi \leq \arg (x) \leq 3 \pi / 2$ :

$$
\text { From } \frac{\partial f}{\partial z}(z)=0 \text { it follows } x=\mathrm{e}^{\mathrm{i} 5 \pi / 4} \text { and } F(x)=2\left((\pi / 4)^{2}+(3 \pi / 4)^{2}\right) \text {. }
$$

4) $x \in S^{1}$ with $\pi \leq \arg (x) \leq 3 \pi / 2$ :

$$
\text { From } \frac{\partial f}{\partial z}(z)=0 \text { it follows } x=\mathrm{e}^{\mathrm{i} 7 \pi / 4} \text { and } F(x)=2\left((\pi / 4)^{2}+(3 \pi / 4)^{2}\right) \text {. }
$$

We conclude that $\mathrm{e}^{\mathrm{i} \pi / 4}, \mathrm{e}^{\mathrm{i} 3 \pi / 4}, \mathrm{e}^{\mathrm{i} 5 \pi / 4}$ and $\mathrm{e}^{\mathrm{i} 7 \pi / 4} 4$ are Barycenters of the set $X$.

Definition 4. (Symmetry propriety SP). Let $\mu$ be a mass distribution on the unit circle. The map $\sigma: S^{1} \rightarrow S^{1}$ is called a symmetry of $\mu$ if: $\sigma$ is an isometry and $\sigma(\mu)=\mu$.

Example 3. $\theta \in[0,2 \pi]$, for which $\theta$ satisfies the map

the symmetry propriety.

$$
A_{\theta}: S^{1} \rightarrow S^{1}, \text { with } A_{\theta}:=\left(\begin{array}{cc}
\cos (\theta) & -\sin (\theta) \\
\sin (\theta) & \cos (\theta)
\end{array}\right)
$$

The mass distribution is defined on two points

$$
\begin{gathered}
x=\left(x_{1}, x_{2}\right), y=\left(y_{1}, y_{2}\right) \text { on } S^{1} . \text { such that } g(x, y)=\pi . \\
g\left(A_{\theta}(x), A_{\theta}(y)\right)=\cos ^{-1}\left(\left\langle A_{\theta}(x), A_{\theta}(y)\right\rangle\right)=\cos ^{-1}(\langle x, y\rangle)=g(x, y)
\end{gathered}
$$

It is clear that for $=\pi, A_{\theta}(x)=y$ and $A_{\theta}(y)=x$. If the distribution is defined over the set of equidistant masses $X=\left\{x_{1}, \cdots, x_{n}\right\}$ then for $\theta=k g\left(x_{i}, x_{j}\right)$ with $A_{\theta}$ is $a \theta$-rotation on the unit circle, with a supplementary propriety that $\forall i=1, \cdots, n, \exists j$ such that $A_{\theta}\left(x_{i}\right)=x_{j} \in X$.

Figure 1 shows some example of symmetry mass points on $S^{1}$. (From left to right) On the first figure, we have two mass points antipodal, the first on $(1,0)$ Cartesian coordinate and the second in $(0,1)$. The second, third and fourth show other types of semmetries.

Theorem 1 (Multiple Barycenter). Let $X=\left\{x_{1}, \cdots, x_{n}\right\}$ on $S^{1}$ be a set of $n$ mass points. If there exists a symmetry map $A_{\theta}$, then $X$ admits a multiple Barycenter.

Proof. For $n=2$ and $\theta=\pi, A_{\theta}$ is the symmetry map such that $A_{\theta}\left(x_{1}\right)=x_{2}$ and $A_{\theta}\left(x_{2}\right)=x_{1}$. Then for all $x \in S^{1}$ hold,

$$
f(x)=g^{2}\left(x_{1}, x\right)+g^{2}\left(x_{2}, x\right)=g^{2}\left(A_{\theta} x_{1}, x\right)+g^{2}\left(A_{\theta} x_{2}, x\right)=g^{2}\left(x_{2}, x\right)+g^{2}\left(x_{1}, x\right)=f(x) .
$$

It follows from our assumptions that $\min f(x)=\min f\left(A_{\theta}\right)$, for all $x \in S^{1}$. It is clear that $A_{\theta} x \neq x$ for all $x \in S^{1}$ and if for all $x^{*}$ is a Barycenter of for all $\left\{x_{1}, x_{2}\right\}$ then $A_{\theta} x^{*}$ is too. In this case we have double Barycenters. Now suppose that $n>2$. Then, for all $x \in S^{1}$, we have

$$
f(x)=\sum_{i=1}^{n} g^{2}\left(x, x_{i}\right) \text { and } f\left(A_{\theta} x\right)=\sum_{i=1}^{n} g^{2}\left(x_{i}, A_{\theta} x\right) \text {. }
$$


Moreover

$$
\sum_{j=1}^{n} g^{2}\left(A_{\theta} x, A_{\theta} x_{j}\right)=\sum_{i=1}^{n} g^{2}\left(x, x_{i}\right)
$$

Thus

$$
f\left(A_{\theta} x\right)=f(x) \text {, for all } x \text { in } S^{1} .
$$

Lemma 3. Under the same assumptions of Theorem 1. Let $k$ be the number of the symmetry maps $A_{\theta}$ in $S^{1}$ which have the propriety $A_{\theta} X=X$, i.e. the distribution of the mass points is $A_{\theta}$-invariant. The number of the multiple Barycenters is $k+1$.

Proof. The proof is left to the reader.

Figure 2 contains three, four, five, six, eight and nine mass points satisfying the symmetry property and non necessary with same weights. The geodesic between two arbitrary and successive mass points is constant. I each of the figures our code generates all possible Barycentes so-called multiple Barycenters.

Figure 3 shows the uniqueness of the Barycenter on $S^{1}$ in the case of four masses without symmetry property.
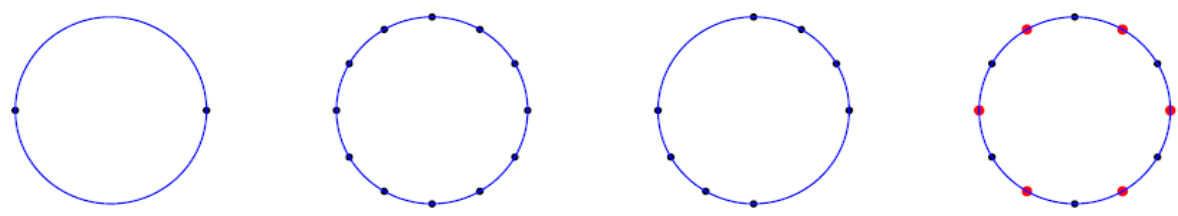

Figure 1. Examples of symmetry masses distribution on $S^{1}$.
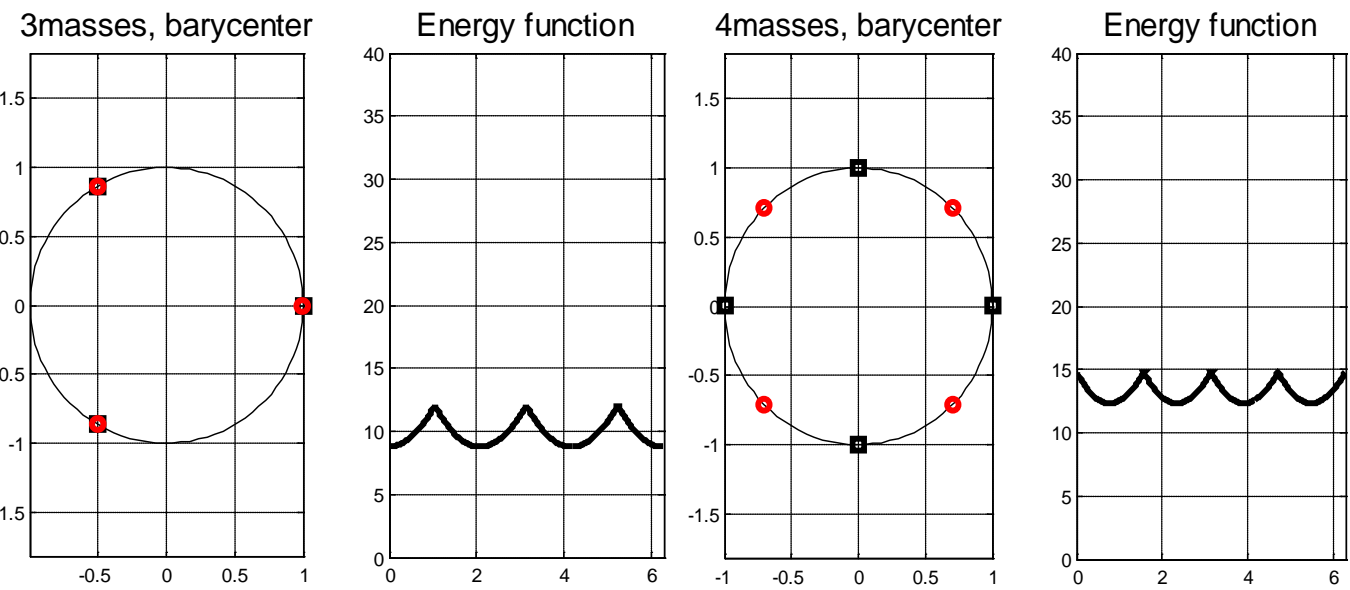

5-masses, barycenter
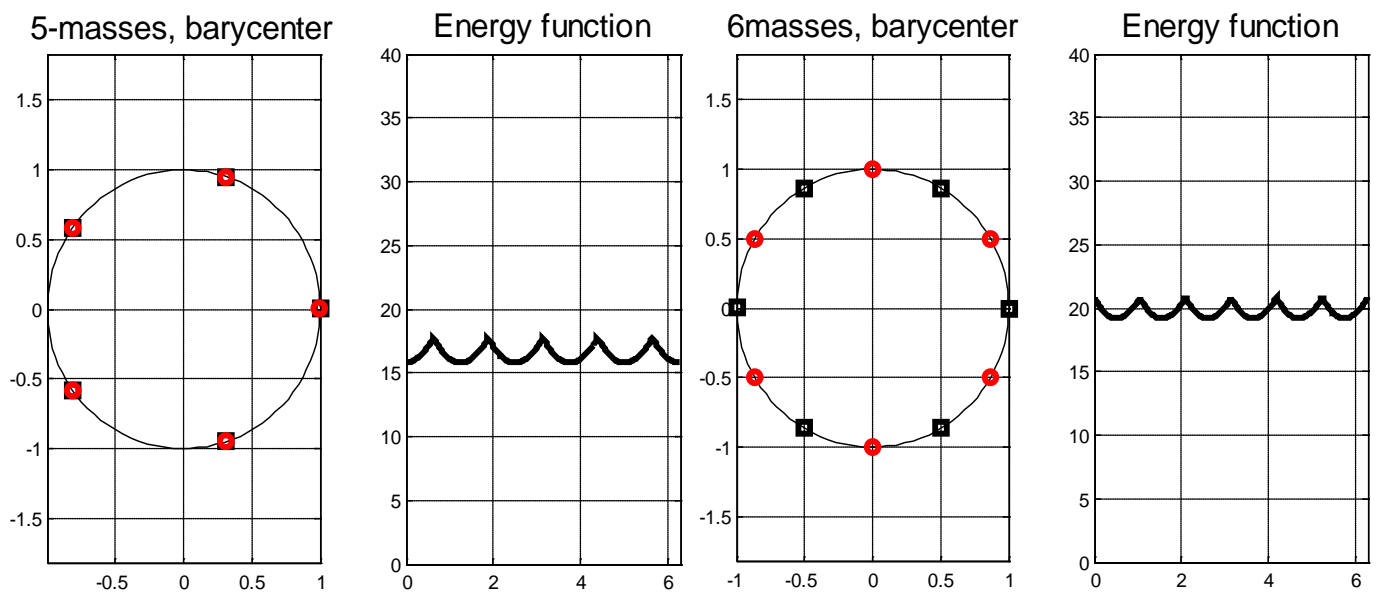

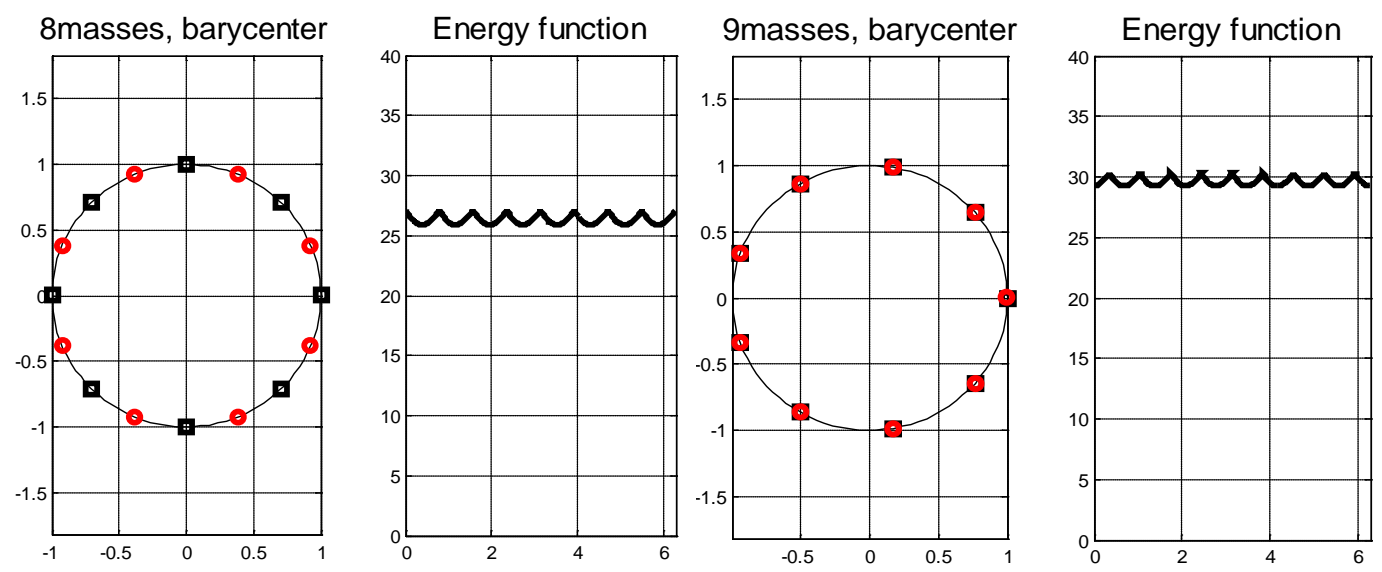

Figure 2. Examples of geodesic multiple Barycenters on $S^{1}$.
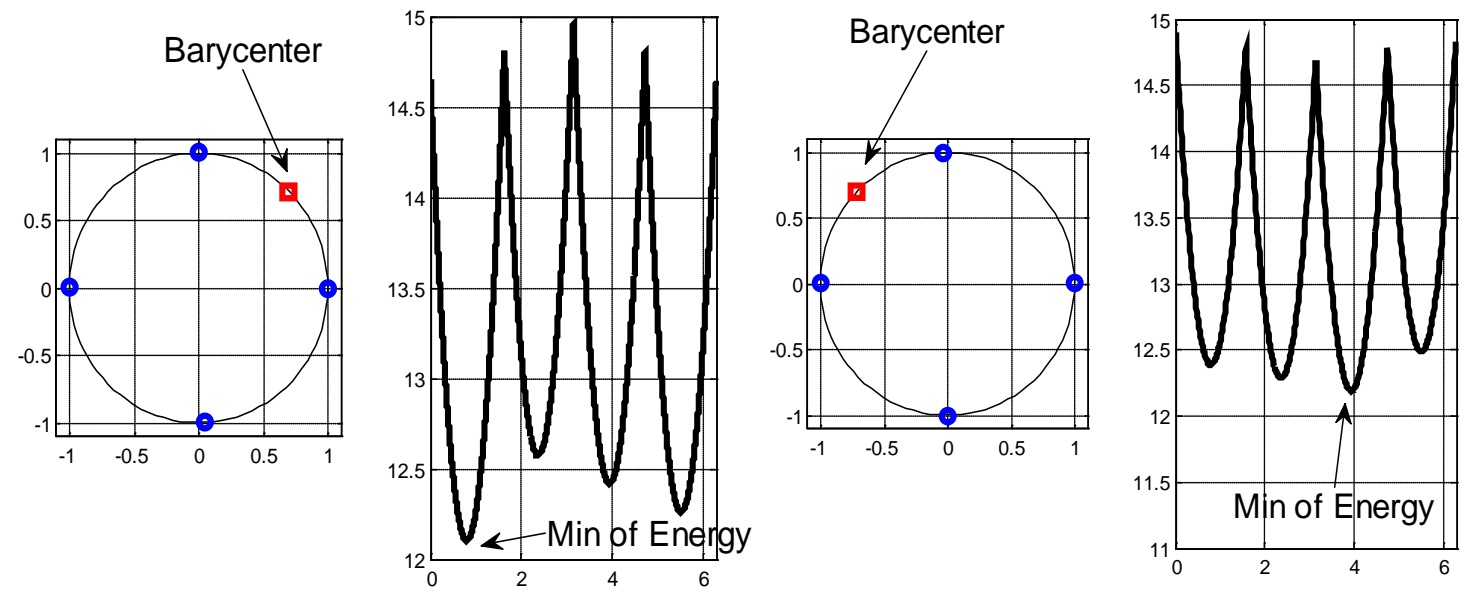

Figure 3. Case of non-symmetry on $S^{1}$ (uniqueness of the Barycenter. (small perturbation of the north and south poles respectively.

\section{Concluding Remarks}

As consequence of the non-uniqueness geodesic between two arbitrary mass points, we have presented an example on the notion of multiple Barycenters. Moreover, we have derived some properties similarly to the Euclidean space. We believe that more interesting cases are to construct significant formulas for arbitrary manifolds.

\section{Acknowledgements}

The authors would like to dedicate this work to Dr. Musaed Al-Subhi Rahimaho Allah.

\section{References}

[1] Le, H.L. (2004) Estimation of Riemannean Barycenters. LMSJ. Mathematics of Computation, 7.

[2] Ruedi, F. and Ruh, E.A. (2006) Barycenter and Maximum Likelihood. Differential Geometry and Its Applications, 24, 660-669. http://dx.doi.org/10.1016/j.difgeo.2006.08.009

[3] Machado, L., Leite, F.S. and Hupner, K. (2006) Riemannean Means as Solution of Variational Problems. LMSJ. Mathematics of Computation, 7.

[4] Ballman, W., Gromov, M. and Schroeder, V. (1985) Manifolds of Nonpositive Curvature. Birkhauser, Boston. http://dx.doi.org/10.1007/978-1-4684-9159-3

[5] Kendall, W.S. (1992) The Propeller: A Counter Example to a Conjectured Criterion for the Existence of Certain Harmonic Functions. Journal of the London Mathematical Society, 46, 364-374. 
[6] Evans, L. and Gariepy, R. (1992) Measure Theory and Fine Properties of Functions. Advanced Mathematical Studies, CRC Press, Boca Raton.

[7] Chen, B.-Y. and Jiang, S. (1995) Inequalities between Volume, Center of Mass, Circumscribed Radius, Order, and Mean Curvature. Bulletin of the Belgian Mathematical Society, 2, 75-86.

[8] Benedetti, R. and Petronio, C. (1992) Lectures on Hyperbolic Geometry. Universitext, Springer-Verlag, Berlin. http://dx.doi.org/10.1007/978-3-642-58158-8

[9] Heintze, E. and Im Hof, H.C. (1977) Geometry of Horospheres. Journal of Differential Geometry, 12, 481-491. 